\title{
APRENDER INGLES con el enfoque comunicativo unido al ESP
}

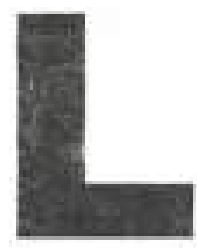

a educacion ha sido un medio de adquisi-

ción de conocimientos desde tiempos inmemorables. Esta se ha convertido en un componente vitai de todo ser humano. y ademas ha sufrido transformaciones a lo largo del tiempo lo cual hace que este en continuo cambio y movimiento

La educacion avanza debido a todos los cambios cientificos y tecnológicos que se presentan a todo nivel y en todo el mundo lo cual ha hecho que ios expertos, investigadores y estudiosos dedicados a profundizar sus conocimientos en un determinado campo del saber, comuniquen, transmitan y compartan dichos avances a los grupos sociales y culturales en general.

Jorge Enrique Jiménez Mónica Naranjo Ruiz

Profesores Departamento de Idiomas Extranjeros Universidad Autónoma de Manizales

El idioma inglés ha sido y segurá siendo un idioma universal, y es un aspecto vital de la educacion, ya que se ha convertido en un 
instrumento de comunicación del saber a todo nivel; por esta razón su aprendizaje se ha constituido en un componente complementario a cualquier disciplina. Teniendo en cuenta que la difusión de la ciencia y la tecnología se transmite, por lo general. en el idioma inglés, las universidades y centros educativos se están viendo en la necesidad de incluir el Inglés como parte del plan de estudios de cualquier Facultad. La Universidad Autónoma de Manizales se ha concientizado de esta necesidad real. La Facultad de Fisioterapia, que ha servido como objeto de estudio de esta investigación descriptiva, hace parte de dicha institución y ha incluido el inglés como componente curricular con el fin de contribuir a la formación integral que exige la sociedad moderna.

Lo anterior ha servido como pauta para investigar los factores negativos y positivos que influyen en el aprendizaje de un idioma con fines comunicativos, pues la adquisición de un idioma debe guiar a la formación humana de los estudiantes, la cual a su vez hace parte de la

\section{El idioma inglés ha sido y seguirá siendo un idioma universal, y es un aspecto vital de la educación}

formación personal y profesional. Teniendo en cuenta lo anterior se ha adoptado el enfoque comunicativo unido al ESP como fundamento de la enseñanza del inglés en la Universidad Autónoma de Manizales.

El enfoque comunicativo surge comoresultado de los minimos logros obtenidos en el denominado \&Situational Language Approach ^ en Gran Bretaña y el «Audio Lingüal Approach en Estados Unidos. Factores como el cambio de las realidades educativas europeas, el crecimiento de la interdependencia y el acercamiento de lo paises Europeos, generó diversas necesidades en todos los campos, facilitando la búsqueda de una nueva onientación de la metodologia de las lenguas extranjeras En Europa en la decada de los setenta Firth y M. A. K Halliday (1957-1978) concentraron sus esfuerzos en el estudio del lenguaje desde un punto de vista comunicativo $y$ funcional, al igual que Dell Hymes (1972). William Labou (1970), Henry Widowson y otros, abogaron tambièn por la necesidad de enfocar la enseñanza de los idiomas hacia el desarrollo 
de la ucompetencia comunicativa* y no hacia el dominio de las estructuras gramaticales

El enfoque acoge la concepción del estudio del lenguaje como un medio de interacción reconoce que las formas gramaticales son componentes importantes de una iengua concentrándose en el componente semántico. La tendencia comunicativa concuerda con la realidad. EI enfoque fomenta actividades y tecnicas para la transmisión de información desconocida de una a otra persona y de selección de ideas y de formas linguisticas apropiadas

La mayor parte de los actos comunicativos tienen un proposito determinado en la vida real, el entoque busca que el estudiante se involucre en cada una de las actividades (aprender haciendo) "Only by practicing communicative activities can we learn to communicates.

Elenfoque considera que el diseño curricular debe tener en cuenta las necesidades
El enfoque considera que el diseño curricular debe tener en cuenta las necesidades comunicativas de los estudiantes comunicativas de los estudiantes con elfin de que ellos sepan que hacen aigo util con el nuevo idioma Para el desarrollo de las diferentes actividades comunicativas se necesita una atmósfera de aprendizaje que promueva el interes la motivación, la seguridad y el respeto a los demas

El enfoque fomenta el uso de textos autenticos y situaciones reaies de la cotidianidad El aspecto cultural es incorporado por medio de actividades comunicativas que hacen referencia al contexto social en que se sucede (dialogos entrevistas. juego de roles. simulacros etc ) tipicos del pueblo de la nueva lengua. De esta forma se valoran más la cultura de la lengua nativa y del idioma extranjero La lengua nativa no es utilizada en el salón de clase $y$ según Windowson (1978) la traducción es una actividad válida especiaimente er la enseñanza de un idioma con propositos especificos

El estudiante en el enfoque es un elemento activo en ei proceso del aprendizaje de la segunda lengua sumaycr 
responsabilidad es la de aprender a comunicarse. El profesor es un facilitador del aprendizaje y desempeña diferentes papeles o roles que varian según las necesidades, los objetivos, las actividades y la planeación.

Los objetivos por los cuales los estudiantes están aprendiendo un idioma son particulares, primordiales y determinan el enfoque y los materiales.

En todo el mundo, los idiomas para objetivos específicos, están creciendo en importancia debido a que muchos estudios muestran que el uso del ESP en diversos contextos es utilizado por científicos, tecnólogos, trabajadores sociales, consejeros agricolas de paises desarrollados, oficiales de inmigración, estudiantes en el extranjero, etc. Tambien este crecimiento se debe al producto de los rápidos cambios tecnológicos y sociales y el incremento de la movilidad de poblaciones, factores caracteristicos de nuestra epoca

Una mayor influencia es el desarrollo de la tendencia de contribuyentes en muchos paises para solicitar una reconsideración de programas de educación a todos los niveles. a la luz de las necesidades nacionales comunes y las posibilidades de las carreras

EI ESP ha llegado al primer lugar porque el inglés es considerado la mejor lengua internacional en ciencia y tecnologia, lo anterior valida el porque se ha implementado el enfoque comunicativo unido al ESP en la Universidad Autónoma de Manizales.

Debido al desfase existente entre la ense- ñanza del idioma en la secundaria y en la universidad, el proceso de enserianza aprendizaje en la universidad se ve afectado por algunos aspectos como la motivación la actitud, el método y el enfoque, los cuales contribuyeron a plantear el fundamento de la investigación ya que cuando se habla de formación el estudiante es el centro.

En la investigación realizada no soio se anaiıo ei proceso ensen̂anza aprenđizaje del inglés lievado a cabo en el aula de clase sino tambien la integración de saberes por parte de los estudiantes y de los docentes en una determinada disciplina Mediante la realizacion de dicha investigación se busca beneficiar tanto a estudiantes como a docente a fin de poder llevar a cabo un proceso enseñanza aprendizaje que satisfaga las necesidades sociales y culturales de los actores

En todo el mundo, los idiomas para objetivos especificos. están creciendo en importancia debido a que muchos estudios muestran que el uso del ESP en diversos contextos es utilizado por cientificos, tecnologos, trabajadores sociales, consejeros agricolas de paises desarrollados 
Durante el desarrollo de la investigación se encontraron varios aspectos que son necesarios en el momento de impartir conocimiento dichos aspectos se abarcaron en dos grandes categorias la pedagogia y la formación, claves para la aplicación del enfoque en el aula de clase.

\section{PEDAGOGIA}

Se puede entender la pedagogía como el puente entre el contexto de la ciencia y los estudiantes. Mediante la pedagogia es posible desarrollar el proceso enseñanza aprendizaje. Para que la enseñanza pueda llevarse a cabo se necesita integrar la ciencia al contexto real del aula de clase

Para llevar a cabo un proceso educativo se requiere de un objeto de estudio. Las cien-

\section{El aspecto sociocultural, el cual es inherente al hombre como ser racional, se en- carga de situarlo en una sociedad que le permite cultivar su intelecto desde distintas perspectivas.}

cias han sido objeto de estudio y fundamentación de los procesos de educación. Se entiende la ciencia como el conocimiento cierto que se tiene de las cosas por sus principios y causas el aula de clase como el espacio que permite la integración del conocimiento y los estudiantes, la enseñanza como el conjunto de conocimientos impartidos por un docente a un grupo de estudiantes.

Para Rafael Flórez la pedagogia describe y comprende los procesos de enseñanza que elevan la calidad de la formación de los individuos, siendo en su proceso de intervencion privilegiado el de la enseñanza de la cultura y de las ciencias.

Como se explicó anteriormente la pedagogia analiza desde todas las perspectivas ei sinnúmero de procesos educativos que guardan una relación directa entre el docente y los estudiantes. Teniendo en cuenta lo anterior, es importante resaltar que todo conocimiento impartido debe tener la capacidad de conceptualizarse y validarse

En la pedagogia se encuentran varios aspectos relevantes a saber cultura, docente y lenguaje.

Cultura: La cultura retroalimenta al hombre continuamente, permitiéndole la producción de conocimiento en una sociedad inmersa en un contexto real sociocuitural Desde el punto de vista educativo, la enseñanza se lleva a cabo en una realidad que debe ser participe en la transformación del pensamiento del hombre a fin de darle un sentido al proceso de enseñanza aprendizaje. 
El aspecto sociocultural, el cuales inherente al hombre como ser racional, se encarga de situarlo en una sociedad que le permite cultivar su intelecto desde distintas perspectivas El hombre como ser racional no puede producir conocimiento de una manera aislada pues es su medio ambiente el que le permite desempeñarse en su realidad

Docente: La educación del nombre en el mundo es la actividad de más importancia y por ende de mayor dificultad ya que múltiples factores que trae consigo la modernidad y el desarrollo cientifico, hacen que se reevalúe el papel del docente en el aula de clase. El docente entonces se convierte en un integrador de conocimiento al aula de clase.

El docente debe desempehar diversos roles según las necesidades y los objetivos propuestos pues es el facilitador del proceso de enseñanza aprendizaje en el cual es investigador y a la vez analista de todas las necesidades e intereses, observador, guia co-comunicador y al mismo tiempo aprendiz de la realidad que io rodea. Debe además tener en cuenta al estudiante como un sujeto activo durante todo el proceso de enseñanza aprendizaje.

Lenguaje: El lenguaje es e! instrumento del cual el docente hace uso para comunicar sentido fuera y dentro del aula de clase

Formación: La formación es considerada como un proceso permanente en todo el ciclo vital del hombre que se sucede al interior de cada individuo y se relaciona con su crecimiento personai intelectual, emocional, valoral y el desarrollo de su autono. mia.

Se apoya externamente en los procesos de educacion y socialización los cuales tienen una función preponderante en la misma. La formación es propia de cada individuo pero se da a partir de la interaccion con el medio Se convierte en un proceso flexible y dinàmico. en constante devenir histöri$c 0$, paralelo e inherente al proceso de desarrollo huma. no y permeable al entorno en el que participan factores
Un aspecto importante en la enseñanza del Inglés en la Universidad Autónoma de Manizales es su contextualizacion a la realidad, la vida social y cultural del individuo 
socioculturales, biológicos e históricos. Segùn Flórez Ochoa, existen cuatro dimensiones indispensables para propiciar la formación del hombre: la universalidad, la autonomia, la inteligencia, la fraternidad o diversidad integrada.

\section{El estudiante como} ser social tiene presente en su vida una serie de necesidades reales, sociales y culturales que lo hacen parte de una comunidad académica
Un aspecto importante en la enseñanza del Inglés en la Universidad Autónoma de Manizales es su contextualización a la realidad, la vida social y cultural del individuo, ésto podria decirse, es el punto de partida para impartir conocimiento. El estudiante como ser social tiene presente en su vida una serie de necesidades reales, sociales y culturales que lo hacen parte de una comunidad académica que debe tenerse en cuenta a la hora de hablar e impartir conocimiento. Es por esta razón que esta investigación debe llevarse a cabo si se pretende obtener buenos resultados en la formación de individuos integrales.

Mediante el uso de instrumentos como la observación y la entrevista se identificó el desempeño de los estudiantes en su desarrollo cientifico y tecnológico en el aula de clase. La investigación confirma la importancia de contextualizar el idioma y de aprenderlo con propósitos especificos.

El trabajo investigativo realizado valida la relevancia del lenguaje y de todas las interrelaciones que éste puede crear cuando se lleva a cabo el proceso enseñanza-aprendizaje de un idioma extranjero.

\section{BIBLIOGRAFIA}

CARVAJAL, Lizardo, Metodologia de la Investigación. Faid Calt 1986 DIAZ, Mario MUÑOZ, José Arturo et all. Pedagogia, Discurso y Poder. Corporación para la Producción y Divulgación de la Ciencia y la Cultura CORPORDIC. Bogotá, Colombia 1997.

FLOREZ, O. Ratael. Hacia una Pedagogia del Conocimiento. McGraw Hill, 1994.

SACRISTAN, J. Gimeno La Pedagogia por Objetivos. De Morata. 1982

WALLANCE ROBINETT, BettY Teaching English to Speakers of Other Languages. St. Paul MN 1978. 


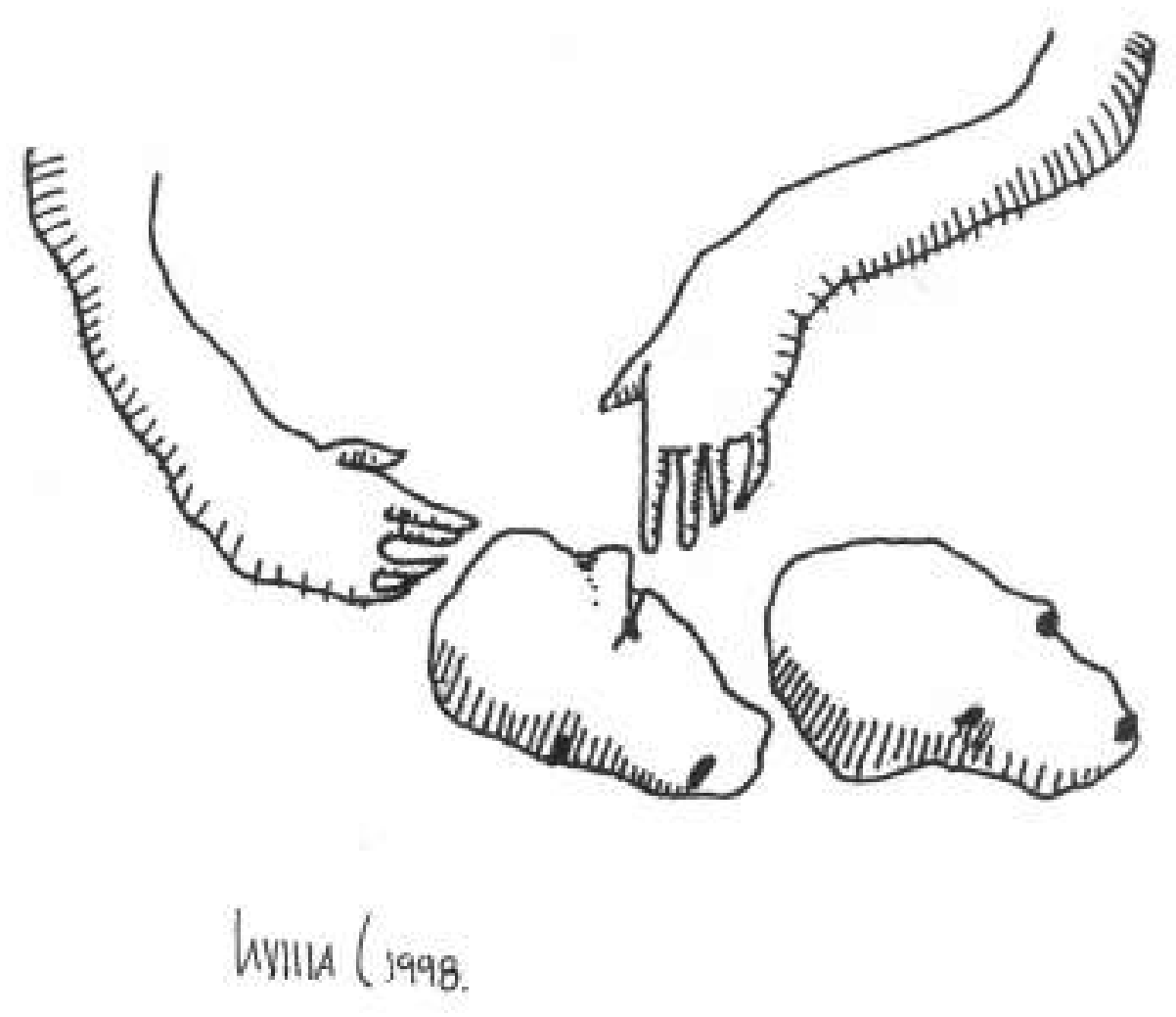

